\title{
Applying System of Systems and Systems Engineering to the Military Modernization Challenge
}

\author{
Kimberly M. Welch \\ Principal Member of Technical Staff \\ Sandia National Laboratories \\ Albuquerque, NM, U.S.A. \\ kmwelch@sandia.gov
}

\author{
Craig R. Lawton \\ Distinguished Member of Technical Staff \\ Sandia National Laboratories \\ Albuquerque, NM, USA \\ crlawto@sandia.gov
}

\begin{abstract}
The challenge of modernizing the current force of military combat systems is one of competing priorities. While planning for future capability requirements and modernization efforts, military acquisition program managers must also consider the immediate demands from the field as the United States is engaged on multiple fronts. These competing demands, along with the challenge of managing large groups of complex systems, motivate the need for an expedient and repeatable systems engineering process. The U.S. Army's Program Executive Office for Ground Combat Systems developed such an approach to support analysis of acquisition programs to meet future capability needs. This paper describes the systems engineering approach and illustrates its usefulness.
\end{abstract}

Keywords: Systems Engineering, System of systems, modeling and simulation, Army modernization.

\section{Introduction}

Defense systems are increasingly complex and interdependent. Addressing capability gaps and future requirements through acquisition requires the ability to look beyond individual system capabilities to a system of systems (SoS) view [1]. Significant work has been done for the Program Executive Office (PEO) for Ground Combat Systems (GCS) to create a systems and SoS engineering approach to address these challenges. The methodology involves a six step process which includes teaming with requirements developers (e.g. combat developers within TRADOC) This process begins with capability gap identification at a high level and maps capabilities down to specific systems that contribute to the overall gap need. Then using appropriate modeling and simulation $(M \& S)$ tools the process continues with quantitative analyses to provide specific measures of performance relative to gaps. The end result is a set of analysis results for a group of systems that tie back to capability gaps for a large force of systems (i.e., brigade combat teams).

\subsection{Overall Systems Engineering Approach}

In order to address the challenges of military modernization, an iterative six step process was developed by the PEO GCS. The premise behind the six step process (Figure 1) was to understand where the greatest gaps in capability exist within the current force and ultimately determine the technology solutions that fill the highest priority gaps, thus providing the greatest performance improvement relative to future capability requirements in the most cost efficient manner.

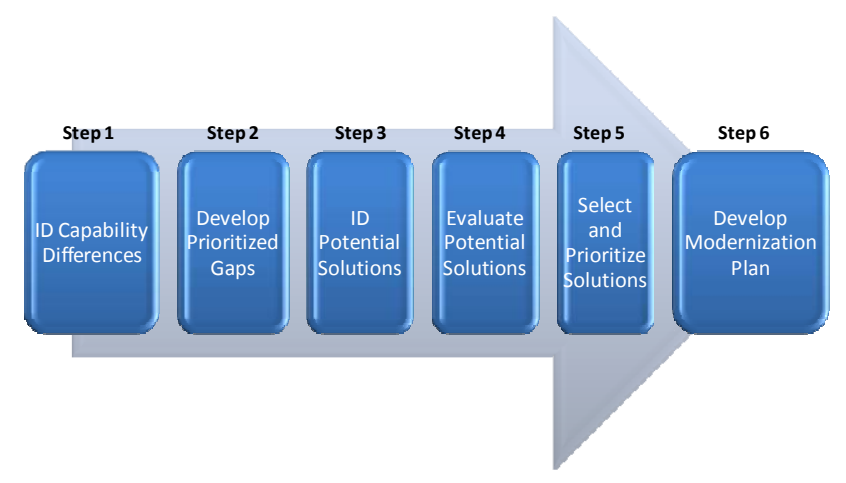

Figure 1- PEO GCS Modernization Assessment Six Step Process

The six step process is used in the context of a Brigade Combat Team (BCT) rather than at a platform level, as is the traditional focus. By focusing at the BCT level, the goal is to measure the effects of technology solutions for modernization that ultimately fulfill the capability needs of a mission or set of missions and not just the performance or functionality of a single platform. To assess the performance at the mission level an understanding of how the mission functions trace to individual system functions and performance is necessary. Figure 2 illustrates this breakdown of mission to the individual platform functions. Defining the relationships in this diagram is essential to supporting analysis at a $\mathrm{SoS}$ level.

[Sandia National Laboratories is a multi-program laboratory managed and operated by Sandia Corporation, a wholly owned subsidiary of Lockheed Martin Corporation, for the U.S. Department of Energy's National Nuclear Security Administration under contract DE-AC0494AL85000.] 


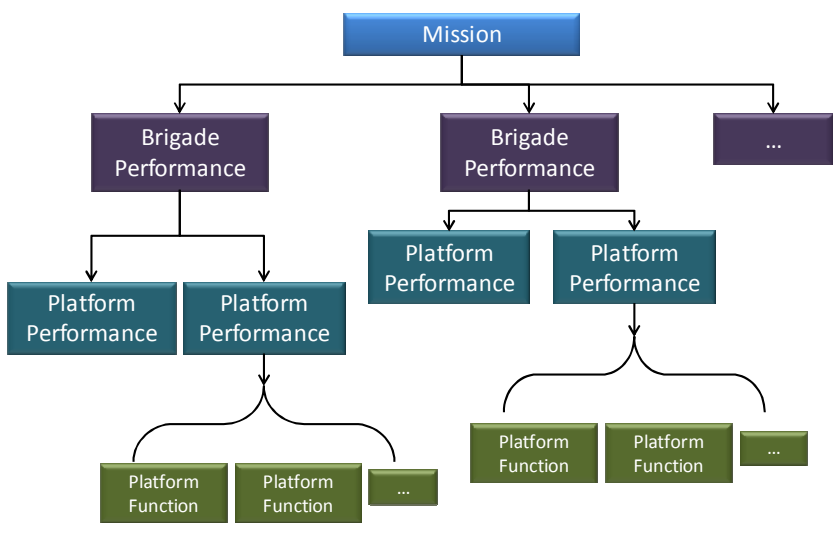

Figure 2 - Mission Decomposition to Platform Functions

In performing the six steps an analysis strategy has been developed leveraging existing work, products and analysis tools where possible, that supports a set of formal, repeatable activities leading to $\mathrm{BCT}$ modernization plans based on best systems engineering practices. This paper will detail the processes as applied to Army Modernization.

\section{Qualitative Decomposition of Army Capability Gaps}

The six step process can be separated into steps that involve qualitative analysis methods and others that depend on quantitative approaches and evaluating metrics. The first three steps primarily utilize qualitative approaches to determine resulting capability gap priorities. This section describes the details of these methods.

\subsection{Parsing gaps into unique simplified gaps}

The first step of the process is to identify capability differences. The approach taken was to define a capability baseline for the current force (CF) that was used to identify and assess the differences between the $\mathrm{CF}$ and known future force $(\mathrm{FF})$ requirements for the operation of future brigades at the SoS, systems and subsystems levels. This task required expertise from personnel within the PEO GCS and information, such as the composition of the BCTs, to support the six step process. This step documented the "as-is" baseline condition of the CF that was used as the comparison measure for all solution and trade study evaluations.

In the second step, the goal is to develop prioritized gaps. This step is the most time consuming, involves several sub-steps and required participation from Army subject matter experts. The development of prioritized gaps began by analyzing the Army-wide materiel gaps contained in U.S. Army Training and Doctrine Command's (TRADOC) 08-13 Capability Needs Assessment (CNA). Upon review of the CNA data, it was apparent that the materiel gaps for many of the required capabilities (RCs) include multiple distinct gap statements and several of the
RCs have identical or very similar gaps. Because of the redundancy, the analytical team created a process to distill the CNA materiel gaps by parsing the statements and elements into distinct parts. These distinct parts are collected, or rolled up, across the RCs to form unique simplified gap statements (termed USGs) around thematically similar "capability areas" as appropriate without the previous redundancy. The unique simplified gap statements served as a better problem definition because they include the functional component as well as all associated descriptive elements. Traceability back to the original materiel gaps and RCs was maintained to facilitate validation by TRADOC personnel. The resulting framework was the backbone for the qualitative analysis.

\subsection{Prioritizing gaps using utility theory}

The unique simplified gaps were presented to BCT subject matter experts within the program manager (PM) and TRADOC capability manager (TCM) communities (in separate sessions) to weight the relative importance of the Unique Capabilities (UCs) to the BCTs. It is important to weight relative importance of UCs based upon required or future planned mission scenarios. Therefore mission scenario descriptions were developed recognizing that the importance of specific capabilities will change depending on the mission involved. Importance values were used and assigned using a scale from 1 to 5 ; 1 reflecting low importance and up to 5 for high importance. A zero value is used to reflect capabilities that have no applicability to the BCT (since CNAs are Army-wide it is possible to have gaps that do not relate to all $\mathrm{BCT}$ missions). The importance factors are normalized across the UCs for each BCT and mission to allow effectiveness scores to be subsequently rolled up to various levels of the analysis framework. It is important to reiterate that this step requires significant involvement from the PM and TCM system experts. Without this involvement the weighting and ranking of UCs cannot be done well.

Once the importance weighting factors were assigned, the BCT's current effectiveness or ability to perform the unique capability was assessed against desired performance. Once again this assessment required system expert participation and opinion. Obviously the more involvement by SMEs, the less biased the scoring will be. The assessment was done using the USG statement and the following 5 to 1 scale:

5 - The ability to perform/provide the unique capability meets objective requirements.

4 - The ability to perform/provide the unique capability meets threshold requirements - the materiel solutions employed within the BCT need only evolutionary improvement or broader fielding to fill remaining gap. 
3 - The ability to perform/provide the unique capability does not meet threshold requirements - supplementary materiel solutions are needed to satisfy the threshold level of performance. These supplementary materiel solutions exist, but require integration into the $\mathrm{BCT}$.

2 - The ability to perform/provide the unique capability does not meet threshold requirements and cannot be adequately improved or supplemented. A revolutionary materiel solution change is needed to replace the existing capability.

1 - There are no materiel solutions present within the BCT to perform/provide this unique capability. A new materiel solution must be developed and incorporated into the BCT.

The effectiveness scores resulting from the second assessment exercise were converted to utility using a nonlinear utility curve.

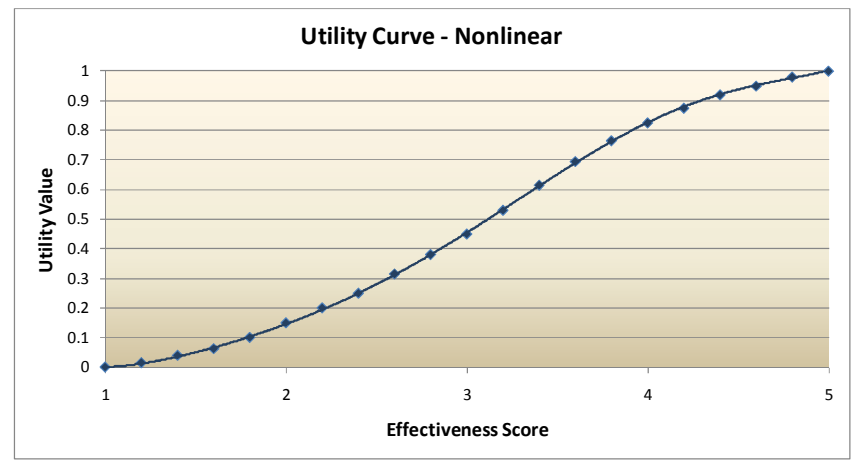

Figure 3 - Nonlinear Utility Curve

The utility value determines how much improvement is possible for a particular USG. As seen in Figure 3, an effectiveness score of 5 converts to perfect utility, or 1, indicating no opportunity for improvement and no capability gap. An effectiveness score of 3 converts to a utility value of 0.45 and indicates an un-weighted gap size of 0.55 (i.e., $1-0.45$; the utility value of achieving objective requirements minus the assessed utility value). The raw gap size value for each UC, if any, was adjusted for importance to the $\mathrm{BCT}$ and mission by multiplying it by its normalized importance factor. In this fashion, a weighted gap size was calculated for every UC and a full baseline assessment was made of the BCT's effectiveness for the described mission(s).

The UCs were sorted based on their weighted gap size (high to low) to create the final prioritized list of gaps. The set of weighted UC utility values, as assessed for the baseline, provided a basis in subsequent process steps to 1) define a starting point for choosing which gaps to assess quantitatively and 2) quantify improvement associated with materiel solutions being proposed to close or narrow the capability gaps.

In step three of the overall process potential solutions were identified for filling the gaps that have now been quantified. Potential solutions, which could include doctrine, organization, and materiel solutions, were identified and associated with the unique capabilities and gaps. Also in this step, an additional qualitative assessment of the BCTs effectiveness to perform a $\mathrm{UC}$ is prepared assuming implementation of identified solution sets. This was done to further prioritize the solutions that were quantitatively evaluated in step four.

Qualitative evaluation of the potential solutions was performed by assessing the expected improvement in BCT mission effectiveness assuming implementation of solutions. This assessment was again performed with $\mathrm{BCT}$ experts providing another set of BCT effectiveness scores.

The set of new scores assumed the solutions are implemented into the BCT. Similar to the baseline assessment discussed previously, each UC's effectiveness score is converted to a utility value and multiplied by the normalized weighting factor reflecting the UC's mission importance. The difference between the UC's baseline weighted utility value and that for the solution case reflects the degree of gap closure associated with that UC. Summing these differences across all of the UCs provides the overall gap closure value, or $\mathrm{BCT}$ effectiveness improvement, associated with the collection of solutions. BCT effectiveness improvement for each solution was calculated by allocating a proportional share of UC effectiveness improvement to the solutions mapped to the $\mathrm{UC}$ and summing across all UCs. Figure 4 and Figure 5 illustrate the results from the qualitative assessment.

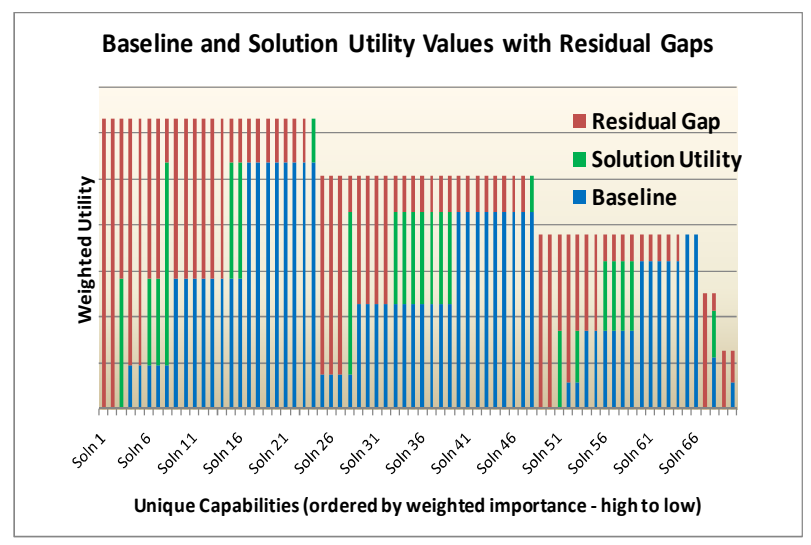

Figure 4 - Example Utility Results from Qualitative Assessment 


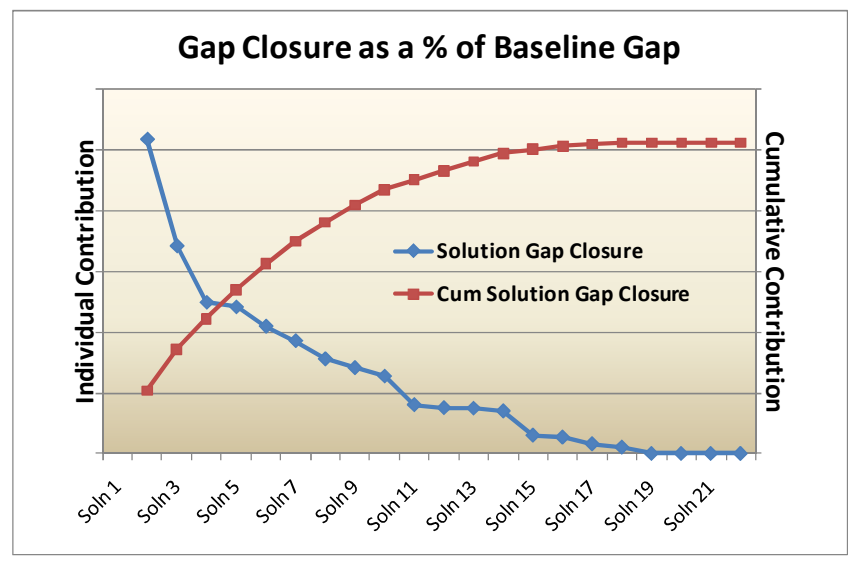

Figure 5 - Example Gap Closure Results from Qualitative Gap Assessment

The results from the qualitative assessment of potential solutions contributed to additional ranking of the capability gaps to be assessed in step four. At this point the identification of quantitative approaches was important to determine how the prioritized gaps and solutions would be assessed.

\section{Quantitative Analysis}

The quantitative processes applied to the six step process provide specific metrics and measures to support the assessment of filling capability gaps. This section describes the tools and approaches used for quantitative assessments as well as the resulting measures of performance that ultimately tie back to capability gaps.

\subsection{Modeling and Simulation (M\&S) tools to support quantitative analysis}

In general, the quantitative analysis is broken down into simulation and optimization. For the purposes of simulation the intent is to evaluate operational effectiveness, within the context of the acquisition community. To evaluate operational effectiveness, simulation and analysis must include not only traditional functional performance variables such as survivability or lethality, but availability variables such as system reliability and operational sustainability (see Figure 6). Only by considering both can a SoS be determined operationally effective.

For the purposes of optimization, the goal is to maximize a systems or SoS operational effectiveness. This results in maximizing performance and availability within the constraints of cost and other limiting factors. The end result allows the user to choose technologies and acquire systems that have the greatest operational effectiveness (e.g., highest performing, most sustainable, most cost efficient).

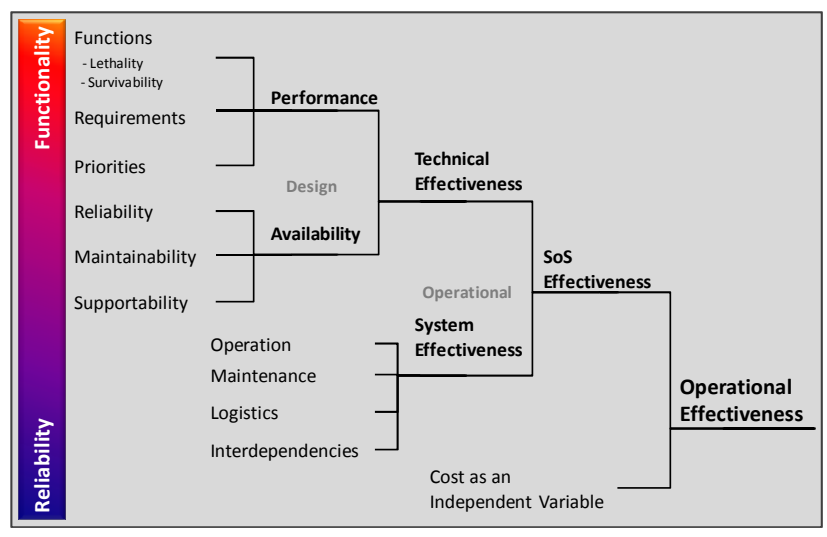

Figure 6- Evaluating Operational Effectiveness using a range of Analyses

No single M\&S tool is capable of performing analysis over the entire spectrum of performance measures that are necessary for evaluating operational effectiveness of a SoS such as a BCT. Therefore the approach has been to develop a framework of tools that can be used independently or collectively (in an integrated fashion). In Figure 6 these are depicted as reliability and functionality tools. Two examples of such tools include the Systems of Systems Analysis Toolset (SoSAT) and the Combined Arms and Support Task Force Evaluation Model (CASTFOREM, currently replaced by the next generation COMBAT XXI), respectively. SoSAT historically has been focused on fleet readiness metrics such as Operational Availability $\left(\mathrm{A}_{\mathrm{o}}\right)$ and Mission Capability (MC) rates; however, it was designed for the DoD to asses SoS performance metrics by representing system interdependencies and evaluating the impact and effects of such interdependencies over a mission. The inclusion of functionality metric results (e.g., survivability, lethality) into the SoSAT model produces metrics such as Combat Availability. CASTFOREM can be used to model performance of certain technology insertions that will improve capabilities such as survivability and SoSAT can be used to analyze logistics and sustainment technologies (e.g. prognostics) and sustainment implications to produce an overall assessment of effectiveness.

As one of the primary tools employed for quantitative analysis, SoSAT was used to construct baseline BCT models prior to evaluating potential solutions. The baseline models are comprised of the entire BCT structure including combat and support systems, sustainment information such as spare part information with associated delay times for spare supply, system reliability information, mission information, and consumables usage and repair services data. The baseline models were used to assess operational availability $\left(\mathrm{A}_{\mathrm{o}}\right)$, sustainment availability $\left(\mathrm{A}_{\mathrm{s}}\right)$ which factors in sustainment constraints, materiel availability $\left(A_{m}\right)$, as well as other sustainment and reliability related metrics. Army subject matter experts assessed the models as valid representations of "as-is" conditions of the BCT 
and thus the models were ready to be used to evaluate potential solutions.

\subsection{Analytical Approach and Results}

Potential solutions to help CF meet FF capability needs come from a number of sources: PM near-term solution sets, Army and industry research and development efforts, and others. As mentioned previously, the identification of potential solutions not only depends upon the qualitative analysis results, but also requires an understanding of quantitative capabilities. (Note: it is plausible that some solutions, whether organizational, doctrinal or materiel, cannot be assessed quantitatively using existing $\mathrm{M} \& \mathrm{~S}$ tools and capabilities. These solutions may be prioritized lower for the quantitative portion of the analysis and need to be assessed by some other qualitative method).

Step four of the overall process is where the majority of the analysis takes place. Using the focused scope defined in step three, an analysis plan for evaluating solutions was developed. The analysis plan began with working meetings with subject matter experts within the BCT PMs and TCMs. The meetings were used to gather relevant and available data regarding the identified solution set. Specific data on solutions and their expected benefits was collected and discussions determined which M\&S tool(s) were appropriate for each solution assessment. Another important aspect of the meetings was the development and definition of the metrics and measures that should be used to evaluate solutions.

Following the working meetings analysts developed study questions and essential elements of analysis (EEAs) that were used to develop model run matrices. Analysts worked with subject matter experts to complete data collection and define and vet analysis assumptions. In cases where multiple models from different tools may be used to address the same study question care was taken to make sure that models were using equivalent assumptions.

Models were run and analysis results generated for each study question and EEA. Figure 7 shows an example of operational availability over mission time results from a SoSAT model comparing a baseline result to several solution alternatives. Statistical results were also available for each analysis and were used to calculate percent improvement (or degradation) as compared to the baseline capability. Where explicit data on solutions was not available, parametric studies were performed to provide a range of possible metric values for a solution set.

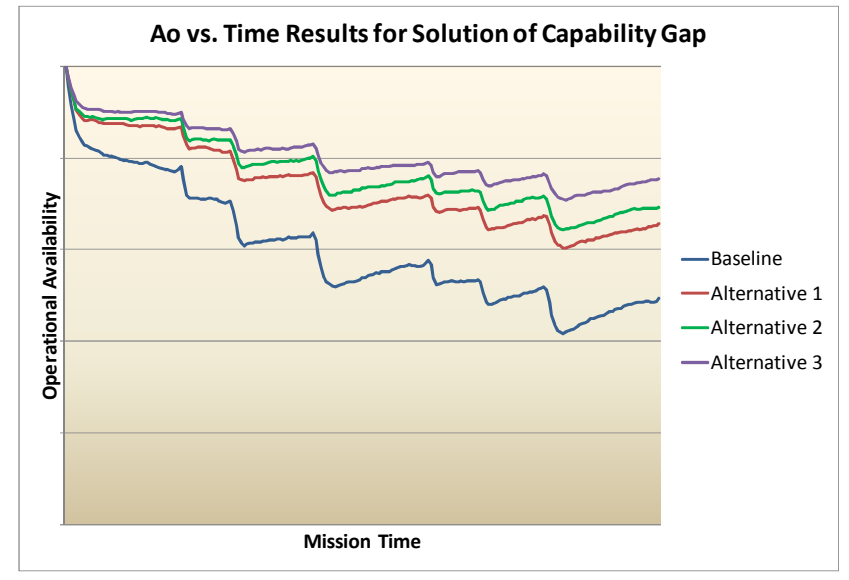

Figure 7 - Example Analysis Results from SoSAT

In some cases multiple models were used to support study question and EEA results. For example, solution sets that directly impact performance in lethality or survivability were analyzed using the CASTFOREM tool. The resulting metrics from this analysis are typically number of shots fired, hits, and kills for specific vehicle configurations as well as number of surviving systems for a battle scenario. This information is beneficial for decision makers, but does not tell the whole story. Using combat damage effects data from CASTFOREM, the SoSAT tool can capture the sustainment and logistic related impacts given the improvement (or degradation) of survivability or lethality for given solutions. Figure 8 shows the resulting combat availability from a SoSAT model that incorporates CASTFOREM results. The combination of these analyses provides more insight into benefits of technology solutions.

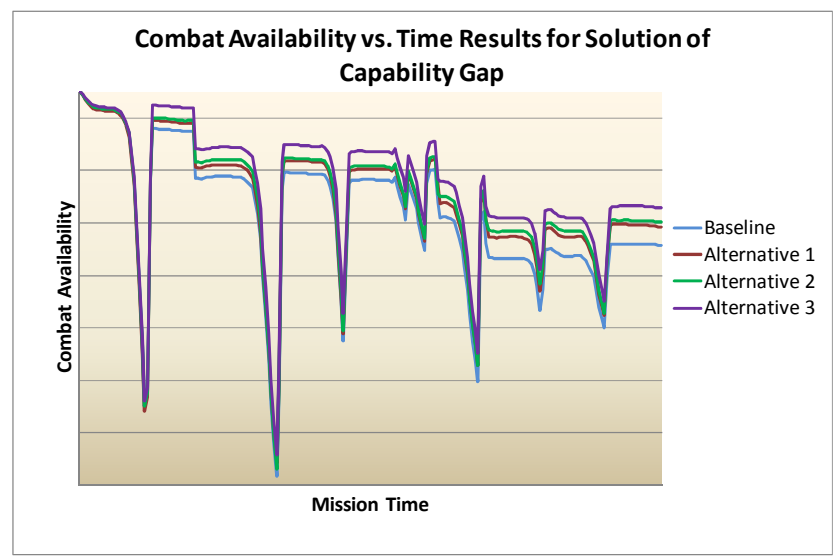

Figure 8 - Example Results from combined CASTFOREM and SoSAT Analysis

Quantitative model analysis results were compiled for all study questions and were provided as input into an optimization approach (discussed in the next section). The quantitative measures support final decisions regarding the potential solution set. The final step is to develop the modernization plan. 


\subsection{Supporting the Modernization Plan}

Final decisions regarding the modernization plan depend upon analysis metrics as well as budget constraints. Keeping in mind that the ultimate goal is to determine how best to transform the CF to meet FF capabilities, step six is most beneficial if a method of optimizing solutions based upon metrics from multiple sources (quantitative, qualitative, and cost variables) is applied.

One such optimization method has been developed for the Army modernization application. This optimization model provides the ability to explore the cost, schedule, and performance trade space and develop an optimized modernization plan. The objectives can be either to maximize SoS performance or minimizing cost over time. These objectives are subject to the following constraints: ability of systems to perform mission roles, the budget(s), the schedule (of upgrading vehicles with solutions), and the mission capabilities defined for each system. Quantitative performance results, qualitative schedule and cost estimates, and other available data feed the optimization approach.

Several different optimization approaches can and should be used for step six. The modeling and analysis infrastructure described in steps one through five is utilized to identify leverage points at the SoS level to provide "best bang for the buck" at the BCT level. The most challenging aspect of the optimization is having the ability to accurately represent the system and SoS constraints. If this can be done the final result is a list of best solutions options for modernizing the $\mathrm{CF}$ to meet SoS level capability needs.

\section{Conclusions}

The Army has a very challenging task of modernizing the $\mathrm{CF}$ to meet capability needs to face future threats. As part of this challenge, the Army must change from a platform-centric view of the world to a SoS view. A repeatable systems engineering process provides high quality analytical support for this challenge. This process depends upon multiple analyses as well as multiple M\&S tools and capabilities. Although the process described in this paper was developed and applied to address real world decisions, we fully expect that it will continue to evolve as more experience is gained.

While the systems engineering approach described in this paper was developed for the problem of Army modernization, it is easy to see how the approach could be used for many problems with similar SoS concepts. Ultimately the goal is to determine the best investment of solution sets to provide the most effective SoS capability. Specifics of the process may change for different problem spaces, but the general concepts remain the same.

\section{References}

[1] Dahmann, Dr. Judith, "Applying Business Process Modeling to Develop Systems Engineering Guidance for New DoD Acquisition Regulations," Proc. NDIA Systems Engineering Conference, San Diego, pp. 2, 5, October 2008.

[2] "Defense Acquisition Guidebook", Defense Acquisition University, March 2011, https://dag.dau.mil.

[3] Verma, Dinesh and Farr, John and Johannesen, Line H., "System Training Metrics and Measures: A Key Operational Effectiveness Imperative", Systems Engineering, Vol. 6, No. 4, pp. 238-241, 2003.

[4] Dahmann, Judith S., Ph.D and Kelley, Mike, "Systems Engineering During the Materiel Solution Analysis and Technology Development Phases", September 2009. 